$\begin{array}{cl}\begin{array}{c}\text { Revue } \\ \text { de Ihistoire }\end{array} & \text { Revue de l'histoire des religions } \\ \text { des religions } & \begin{array}{l}\mathbf{3} \mathbf{2 0 0 7} \\ \text { Varia }\end{array}\end{array}$

\title{
Être nazir : du guerrier yahwiste au vœu cultuel du judaïsme ancien
}

Origine et transformation d'un rite de cheveux

To be a nazir: from the yahwistic warrior to the worship vow of the Ancient Judaism. Origin and transformation of a hair ritual

\section{Christophe Lemardelé}

\section{(2) OpenEdition}

Journals

Édition électronique

URL : http://journals.openedition.org/rhr/5305

DOI : 10.4000/rhr.5305

ISSN : 2105-2573

Éditeur

Armand Colin

Édition imprimée

Date de publication : 1 septembre 2007

Pagination : 275-288

ISBN : 978-2200-92334-1

ISSN : 0035-1423

Référence électronique

Christophe Lemardelé, "Être nazir : du guerrier yahwiste au vœu cultuel du judaïsme ancien », Revue de l'histoire des religions [En ligne], 3 | 2007, mis en ligne le 01 septembre 2010, consulté le 21 avril 2019. URL : http://journals.openedition.org/rhr/5305; DOI : 10.4000/rhr.5305 


\title{
CHRISTOPHE LEMARDELÉ
}

Laboratoire des Études sémitiques anciennes

(CNRS-Collège de France-Paris IV)

\section{Être nazir : du guerrier yahwiste au vou cultuel du judaïsme ancien Origine et transformation d'un rite de cheveux*}

\begin{abstract}
Il n'y eut pas un rite de nazir mais deux! Le mieux connu est le vœu prononcé en vue d'un bienfait divin qui fut élaboré à partir de matériaux anciens (les interdits) par une élite sacerdotale à l'époque perse. Ce vœu fut pratiqué sans évolution décelable à l'époque hellénistique et à l'époque romaine. L'autre nazir est bien plus difficile à déterminer. Les textes bibliques le mentionnant ont été pour la plupart retravaillés, perdant ainsi quelque peu sa signification. C'est l'aspect le plus étonnant du sujet puisqu'il nous fait remonter aux époques les plus anciennes du "yahwisme » des «Israélites». Avant de ne devenir qu'une survivance au sein des appareils étatiques progressivement mis en place dans le royaume du nord puis dans celui du sud, il fut sans doute un rite de jeunes hommes sous l'égide d'un dieu guerrier (Yahweh).
\end{abstract}

\section{To be a nazir: from the yahwistic warrior to the worship vow of the Ancient Judaism. Origin and transformation of a hair ritual}

There was not one rite of nazir but two! The one well-known is the vow pronounced in order to have a divine profit. It was elaborated with ancient elements (the prohibitions) by a priestly elite at the persian period. This vow didn't know any evolution at the hellenistic period and at the roman period. The other nazir is more problematic. The biblical texts, referring to it, have been mostly changed as time goes on, losing somewhat its meaning. This is the most surprising aspect of the subject because, with it, we go back to the ancient periods of the "Israelites' yahwism". Before becoming a survival in the states progressively established in the northern kingdom then in the southern, it was probably a rite for the young men under the aegis of a god warrior (Yahweh).

\footnotetext{
* Thèse de doctorat d'histoire des religions, sous la direction de $\mathrm{M}^{\mathrm{me}}$ Hedwige RouillardBonraisin, présentée à l'École pratique des Hautes Études, Ve section Sciences religieuses, le 3 février 2007 (1 vol., 400 p.) - Membres du jury : M ${ }^{\text {mes }}$ et MM. les professeurs Philippe Abadie (Institut catholique de Lyon), Jean-Marie Husser (Université Marc Bloch de Strasbourg), Renée Koch-Piettre (EPHE, Ve section), Philippe de Robert (Faculté de théologie protestante de Strasbourg), Hedwige Rouillard-Bonraisin (EPHE, Ve section), Francis Schmidt (EPHE, Ve section). - (Mention très honorable.)
} 
Le sujet s'imposait presque naturellement dans une recherche d'ensemble sur les rites du judaïsme ancien. Mais il s'imposait plus encore du fait qu'il n'avait pas été étudié très précisément. Le manque de données et la difficulté à les interpréter - c'est un sujet presque exclusivement biblique! - ont pu décourager quelques chercheurs. Il s'agissait donc de dépasser la simple monographie se contentant de paraphraser les sources tout en évitant d'aller trop audelà d'elles par une unique approche anthropologique. La dimension historique du sujet était à prendre impérativement en compte car elle était à même d'expliquer les incohérences suggérées par les sources : comment concilier le vœu de nazir du Pentateuque (Nombres 6) et le héros nazir du livre des Juges (chapitres 13 à 16) ? L'historiographie sur le sujet montre à quel point les auteurs n'ont pas suffisamment prêté attention à cette dimension qui permet de voir qu'il y eut évolution du rite, et non pas tant une évolution « naturelle » qu'une transformation. Vouloir trouver le vœu de nazir dans les mentions les plus anciennes conduisait immédiatement à l'impasse. De même, opposer une consécration sans condition et à vie à un vœu conditionnel et temporaire ne permettait pas de résoudre les difficultés. Certes, cette approche se faisait plus diachronique mais non dénuée de présupposés : le nazir à vie aurait reflété la religion israélite spontanée et sincère des prophètes opposés au rituel détaillé et réglementé du judaïsme sacrificiel. Nous devions donc partir des textes en les étudiant en détail, en se gardant de toute interprétation succincte, tout en les situant chronologiquement le mieux possible afin de pouvoir les comparer entre eux avec profit.

Le texte de référence se trouve être Nombres 6. L'erreur de beaucoup d'auteurs est de considérer ce texte comme étant celui où le rite de nazir est explicité. Or il n'est qu'un texte où un rite de nazir est décrit. Ainsi tout expliquer à partir de lui amène à faire des erreurs d'interprétation, qui plus est, à faire acte d'anachronisme. Les textes législatifs du Pentateuque sont bien délicats à dater mais la critique interne des textes, déjà très au point à la fin du XIX siècle, permet de les situer, pour l'essentiel, après la chute de Jérusalem et l'exil des élites du royaume de Juda à Babylone, soit après le VI siècle av. J.-C. En outre, ceux contenus dans le livre des Nombres doivent 
être parmi les plus récents étant donné l'aspect composite du livre - la constitution du Pentateuque s'est probablement close avec l'élaboration de ce livre. Le rite décrit se situe donc dans les dernières étapes d'un processus de «construction » religieuse, soit vraisemblablement au IV siècle. Si l'on met en avant l'idée de monothéisme pour spécifier le renouveau religieux de cette période, on oublie en général le «dogme » mis en place et qui est clairement défini en Lévitique 10, 10 : séparation du sacré et du profane, de l'impur du pur. Le rite détaillé en Nombres 6 est clairement marqué par cette idéologie : le nazir devenait sacré et, à ce titre, était contraint à la plus grande pureté. Mais la logique du système établi eût voulu qu'il fût situé à un rang proche des prêtres et du grand prêtre. Rien de tel dans les faits! Le rite de nazir était surtout l'objet d'un vœu traditionnel, l'« ascétisme » n'était pas la finalité du vœu mais la condition pour obtenir un bienfait divin.

L'insertion d'un rite de nazir dans le nouveau système cultuel a entraîné également la mise en place de prescriptions sacrificielles complexes en fin de vœu : le nazir devait trois sacrifices d'ovins à la fin de sa période, un sacrifice de purification associé à un holocauste, et un sacrifice partagé conduisant à un repas. En outre, l'impureté la plus grave aux yeux des auteurs de ce système - l'impureté par un cadavre - les a conduits à élaborer une procédure de purification, complexe également à cause des sacrifices requis - un sacrifice de purification et un holocauste d'oiseaux, et un ovin en réparation et à cause de son rattachement sans doute ultérieur à la procédure de Nombres 19 : le sacrifice d'une vache rousse pour obtenir de l'eau lustrale. Bref, ce qui subsiste d' « ancien » dans ce rite lorsqu' on le compare aux autres données est restreint, ce sont seulement les deux interdits énoncés au début du texte dans cet ordre : l'interdit de vin et d'alcool (ou de bière) et l'interdit de se couper les cheveux - la nécessité d'être pur est fluctuante selon les textes.

L'autre texte de référence d'un rite de nazir est très différent. Mais il comporte une liste d'interdits assez semblable. Il s'agit du récit des exploits de Samson tant sujet à controverses. En effet, le personnage est rarement compris car il n'est appréhendé qu'en tant que personnage biblique et le récit est bien souvent lu comme seule 
transition ironique entre des juges plus constants et vraisemblables et les personnages de Samuel et de David. Cette lecture succincte et orientée du récit de Samson se fait dans le cadre d'une histoire deutéronomiste élaborée après la fin de la royauté en Juda, donc après le $\mathrm{VII}^{\mathrm{e}}$ siècle, et le rite de nazir est alors compris comme étant un des éléments intégrés au récit fort tardivement: les auteurs de cette histoire construite d'Israël auraient voulu expliquer le topos des cheveux longs de Samson par le vœu de nazir. Cependant, lorsque l'on compare strictement Juges 13 à Nombres 6, l'énoncé des interdits est différent (ordre et vocabulaire). Surtout, Samson pas plus que sa mère pour la conception de celui-ci ne prononcent un vœu ! Il faut ajouter que le texte hébraïque ne comporte pas la mention d'une quelconque sacralité du nazir. Le rite évoqué dans ce récit précède donc le vœu sacerdotal et se rapporte plus vraisemblablement à la nature guerrière du héros. Samson le dit lui-même à Dalila au chapitre 16 : sa force lui vient de là. Ainsi, le motif du nazir, même très littéraire, du texte des Juges ne correspond en rien à la finalité du rite votif détaillé dans le livre des Nombres. Certes, penser, dans une perspective toute narrative, que le nazir puisse être intrinsèquement lié au récit n'est pas non plus satisfaisant car les fautes du héros ne correspondent pas exactement aux interdits énoncés en préambule. Une interprétation nuancée permet d'affirmer que les interdits se sont appliqués aux fautes lors de l'adaptation du récit : le nazir n'est ni à l'origine du récit ni sa dernière révision mais est probablement intervenu lors d'une relecture yahwiste de la geste d'un géant dont le nom fait référence au dieu solaire Shamash. Cette relecture a quelque peu transformé le géant en jeune homme sous la tutelle de Yahweh.

Le dernier grand texte pouvant trancher la question entre un rite de nazir guerrier et un simple vœu s'avère malheureusement difficile d'emploi. Il y a en effet un problème textuel en 1 Samuel 1 et un problème littéraire : Samuel est dit nazir dans un récit de conception proche de celui de Samson mais pas dans toutes les versions ni aux mêmes versets, et ce titre est surprenant pour un prophète. Ce sont les versions de la Septante et celle d'un manuscrit de Qumrân qui attestent que Samuel fut nazir. Ce point de critique textuelle est renforcé par le témoignage de Flavius Josèphe qui traite de Samuel 
comme il l'a fait de Samson : pour lui, ils étaient tous deux prophètes, ne buvant que de l'eau. Le titre de prophète a de quoi surprendre pour Samson mais l'historien juif voulait se montrer cohérent car, venant de définir le vœu de nazir d'après Nombres 6 dans le livre IV de ses Antiquités juives, il ne pouvait insérer un titre sans lien avec une pratique votive dans le livre V. Enfin la version hébraïque fragmentaire d'un livre de sagesse du $\mathrm{II}^{\mathrm{e}}$ siècle av. J.-C. (Siracide ou livre de Ben Sira) - non considéré comme sacré dans le judaïsme mais retenu dans le canon catholique - apporte la preuve que Samuel était nazir dans les textes à cette époque-là ; le petit-fils de l'auteur, traduisant l'œuvre en grec, n'ayant pas jugé utile de reprendre ce titre.

Mais la présence du terme pour Samuel pose une difficulté plus grande que le problème textuel. En effet, cela attesterait une troisième forme de nazir concernant les prophètes. Toutefois, Samuel est un personnage qui cumule les titres et les fonctions (prophète, prêtre, juge) ce qui fait douter de son historicité, qu'il soit nazir n'a pas plus de sens que d'être juge et même prophète : son rôle tout littéraire est d'oindre les rois d'Israël, Saül puis David. Surtout, la difficulté se trouve résolue lorsque l'on restitue le texte originel en remplaçant Samuel par Saül. Car le texte est plus cohérent ainsi puisque la naissance d'un enfant nommé Saül et non Samuel est clairement annoncée. Tout le texte tourne autour du vocabulaire du don et de la demande : l'enfant demandé sera donné nazir au Dieu d'Israël. Nazir est traduit par doton dans la Septante et «demandé » se dit shâ'ûl, alors que Samuel signifie « son nom est El». Il est assez clair qu'il y a eu une substitution de noms : « elle [Hannah] l'appela du nom de Samuel car [dit-elle] de Yahweh je l'ai demandé » (1Samuel 1, 20). Que Saül fut nazir est bien plus acceptable étant donné que l'histoire du premier roi d'Israël comporte des aspects légendaires étrangement proches du récit mythique de Juges 13-16. Le premier roi d'Israël est en effet l'auteur en 1Samuel 11 d'un exploit comparable aux exploits de Samson : la narine brûlant de colère et possédé par l'esprit de Yahweh, Saül dépèce en morceaux une paire de bœufs. La mention de l'esprit divin en lien avec un exploit est même présente au chapitre précédent mais les incohérences nombreuses dans un passage du récit semblent prouver qu'il y eut réécriture du passage, cela afin de 
faire de Saül, devant le peuple, le roi choisi par Yahweh. Toutefois, l'exploit n'est pas perdu car on le retrouve maladroitement attribué au fils du roi, Jonathan, en 1Samuel 13, 3. Mais c'est bien Saül qui souffle dans le shophar pour rassembler les Hébreux et il est dit au verset suivant : "Saül a frappé la garnison des Philistins ». Or l'action se situe à Gibea, ville de garnison philistine où devait se rendre Saül dès le chapitre 10, avant d'être « détourné » par des rédacteurs ultérieurs, initialement vers Gilgal puis finalement vers Miçpa.

Il en résulte que le premier chapitre du livre de Samuel est bien une source sur le nazir, comparable à Juges 13. À la différence de ce texte, le récit de conception du futur roi comporte bien un vœu, mais il s'agit d'un rite qui ne se rattache pas directement au rite de Nombres 6. Hannah supplie Yahweh des Armées dans le temple de Shilo pour surmonter sa stérilité et promet de donner l'enfant comme nazir en contrepartie : elle ne prononce donc pas, qui plus est pas pour elle-même, un vœu de nazir ! L'absence d'un tel vœu, comme en Juges 13, confirme donc l'existence d'un rite originel non votif. Et dans cette conception, nous n'avons pas de sacralité du nazir, pas plus que l'impératif de pureté.

Ne pouvant nous contenter de ces textes difficiles à utiliser, nous avons étudié les quelques mentions de nazir présentes dans les textes hébraïques de la Bible, réputées assez anciennes et plus faciles à dater pour certaines d'entre elles. Qu'il s'agisse de Deutéronome 33, 16 (VIII ${ }^{\mathrm{e}}$ siècle), d'Amos 2, 11-12 (VIII ${ }^{\mathrm{e}}$ siècle), de Jérémie 7, 29 (fin $\mathrm{VII}^{\mathrm{e}}$ siècle) ou de Lamentations 4, 7-8 ( $\mathrm{VI}^{\mathrm{e}}$ siècle), aucune de ces mentions ne fait état d'un vœu de nazir et presque toutes signalent un rite pratiqué par des jeunes hommes. La brève mention d'Amos 2 nous a permis même d'établir un lien plus net entre le nazir et l'esprit de Yahweh possédant Samson et Saül. En effet, ce texte dit que Yahweh faisait lever des prophètes et des nazirs. Or c'est pénétrés d'esprit divin que les premiers prophétisaient. Les prophètes (nebî'îm), qu'il importe de distinguer des «prophètes écrivains » ne portant d'ailleurs pas ce titre, apparaissent bien souvent dans les textes comme entrant en transe. Les nazirs pouvaient leur être adjoints si l'on se fie aux récits concernant Samson et Saül car la possession 
divine faisait qu'ils devenaient furieux. Or la mention d'une telle possession dans sa formulation la plus archaïque en lien avec un exploit n'est présente que pour ces deux héros. L'évocation poétique qui se trouve en Deutéronome 33, 16-17 suggère également une telle interprétation puisque la faveur de Yahweh venant sur la tête de l'élu lui donne la force du buffle. Il nous est donc apparu, d'une part, que les personnages de Samson et de Saül avaient été pour des raisons diverses nazirs afin de rendre compte de leur force en fonction de Yahweh ; d'autre part, sans qu'il soit possible d'avoir des précisions tant rituelles que chronologiques, qu'il y avait eu une pratique du nazir très différente de celle énoncée en Nombres 6, ne concernant que les jeunes hommes, expliquant et stimulant leur force sauvage, cela peut-être aux temps des monarchies israélite et judahite et, plus probablement, avant même ces entités étatiques. Un tel rite ou une telle institution a dû en effet être vivace dans une société non étatique, subsistant au temps des deux royaumes mais perdant probablement sa nécessité, comme le suggérait déjà Max Weber. Les dernières mentions de Jérémie 7 et de Lamentations 4 font d'ailleurs état d'une institution reliquat, l'une faisant de Jérusalem la nazir de Yahweh, devant couper sa belle chevelure consacrée en signe de deuil pour la destruction à venir ; l'autre décrivant de manière contrastée les jeunes hommes nazirs avant et après la destruction de la cité par les Babyloniens, ayant donc survécu aux combats.

Après avoir fait la synthèse de toutes ces analyses, nous avons tenté d'aller un peu plus loin en étudiant la fonction guerrière israélite telle qu'elle apparaît en filigrane dans la Bible hébraïque. Malheureusement, même s'il existe des textes pouvant refléter des pratiques guerrières anciennes, «tribales », leur édition fut beaucoup plus tardive et les liens avec le nazir sont presque impossibles à déceler. Le seul élément à peu près certain est que le nazir concernait des jeunes guerriers (bachûrîm) et non de grands guerriers confirmés ( gibbôrîm), bien que ces derniers pussent être des nezîrîm pour accéder à ce statut d'homme fort. La recherche étymologique, se basant sur la récente théorie de Georges Bohas privilégiant l'étymon à deux consonnes comme racine effective, nous permit de bien différencier les racines $n z r$ et $n d r$, le nazir n'étant pas un «voué » mais, plus 
précisément, un «séparé », et ceci dans un sens moins positif que celui de «consacré ». Au contraire, sans doute devait-il être perçu comme étant inapprochable du fait de la présence de l'esprit divin en lui ; présence comparable à celle du menos divin placé dans la poitrine des guerriers de l'Iliade. C'est le texte de Nombres 6, réélaborant le rite, qui donne ces significations et, finalement, brouille les cartes en reliant le nazir à la prononciation d'un vœu (nédèr) et en faisant de celui qui faisait un tel vœu un personnage "sacré » (qâdosh) dans le système sacerdotal déterminant tout en fonction de l'opposition stricte impur/sacré.

La première partie de notre recherche s'est donc achevée sur une thèse simple et malheureusement imprécise : le nazir devait être un jeune guerrier d'exception, s'abstenant de vin et gardant la chevelure longue, possédé par un esprit divin le menant à accomplir des exploits étonnants, cela à un moment où des clans et des tribus n'étaient pas encore intégrés dans une structure étatique, soit avant les $X^{\mathrm{e}}$-IX $\mathrm{X}^{\mathrm{e}}$ siècles. Au VIIII siècle, le prophète Amos mentionne encore le rite mais comme s'il était corrompu. Au vie siècle, les Lamentations ne nous donnent plus à voir que des nazirs à l'écart des combats. Le terme et le rite auraient dû disparaître dès la fin du «premier temple » s'il n'y avait eu la renaissance religieuse dont font foi les textes législatifs du Pentateuque.

$$
* * *
$$

La seconde partie de notre étude a été consacrée à l'analyse exclusive du vœu de nazir. En reprenant le texte de Nombres 6, ses versions araméennes et quelques sources grecques, nous avons pu éclaircir certaines obscurités présentes dans le texte hébraïque. Par exemple, les targums et la Mishna précisent que les cheveux rasés à la fin du temps de nazir n'étaient pas offerts sur l'autel des sacrifices mais seulement brûlés dans le feu de la marmite où cuisaient les viandes sacrificielles. Ainsi la chevelure n'était pas sacrifiée mais seulement détruite. Mais l'étude des versions nous a surtout permis de voir que la traduction grecque de la Septante avait été interprétative, le vœu de séparation devenant un grand vœu de purification. 
Ceci explique pourquoi certains auteurs, dépendant de cette traduction, ont pu s'écarter de la signification et du rite même de nazir.

Philon d'Alexandrie s'est appuyé sur la traduction grecque du verbe pâlâ' au factitif - «rendre grand un vœu »-pour faire «le grand vœu », classé avec les sacrifices dans le premier livre de ses Lois Spéciales et non dans le second où il traite pourtant des vœux et des serments. Pour le philosophe alexandrin, ce rite était le meilleur sacrifice, «preuve d'amour pour Dieu » puisque l'on s'offrait soimême. Mais, afin de gommer les aspects violents du sacrifice, il précise que la coupe des cheveux permettait d'éviter que l'autel sacré ne fût souillé de sang. Comprenant bien que la chevelure était consumée dans le feu de la cuisine, Philon en vient à affirmer que le feu culinaire était également sacré pour sauvegarder sa lecture sacrificielle orientée vers le don. Quant à l'interpolateur d'un passage des Actes des Apôtres (21, 23-27), introduisant quatre «nazirs» dans un récit où Paul était à l'origine seul à se rendre au temple de Jérusalem, il s'est mépris en comprenant, d'après la version grecque, l'accomplissement de vœux comme étant une procédure de purification ; ce qui retire toute qualité historique à ce bref épisode, l'interpolateur ayant voulu accentuer la piété halakhique de Paul et ainsi montrer à quel point son arrestation était injuste.

La pratique du vœu est confirmée par beaucoup de témoignages de l'époque romaine. Le recoupement de témoignages émanant de Flavius Josèphe et d'écrits rabbiniques (Mishna) a permis aussi de préciser certains détails comme la durée habituelle du temps de nazir - un temps relativement court ne permettant pas d'avoir une chevelure longue : trente jours - et de déterminer le motif du vœu : une situation de détresse. Malgré l'exemple épigraphique d'un certain Jonathan portant le surnom de nazir, sous-entendant qu'il le fut un temps très long, nous n'avons trouvé aucune interprétation pratique - et non spéculative comme celle de Philon - du rite allant vers l'ascétisme. Le traité de la Mishna qui lui est consacré montre au contraire qu'il ne différait pas des autres vœux, les rabbins discutant longuement au sujet de sa seule prononciation afin d'établir la validité du vœu ; cela d'après une conception des vœux toute pharisienne : l'objet voué (qorbân) n'étant plus une offrande mais quelque chose 
de consacré et donc d'interdit. Cette conception est très critiquée dans un écrit sectaire de Qumrân (Document de Damas). Si l'on accepte que les auteurs de ces textes étaient des Esséniens, alors la critique se comprend car ces derniers, quand ils prononçaient des vœux, faisaient bien parvenir leurs offrandes votives au temple de Jérusalem d'après Flavius Josèphe. Cette pratique classique des vœux, structurée en trois temps : demande/promesse, exaucement, acquittement, ne signifie pas que le vœu de nazir était un simple contrat avec la divinité, il était au contraire effectué avec une grande piété puisqu'il s'agissait de reconnaître la supériorité et l'unicité du dieu de Jérusalem en lui faisant des vœux, qui plus est un tel vœu exigeant un engagement conséquent. Ainsi, excepté pour les Pharisiens, la pratique des vœux dans le judaïsme était commune à la pratique des vœux dans le bassin méditerranéen à cette époque. Même si nous n'avons qu'un seul ex-voto, qui plus est obscur et trouvé en Galilée (Tel Dan), le vœu invoquant le dieu de Jérusalem différait peu structurellement d'un vœu phénicien ou grec. Sa prononciation pouvait être accompagnée d'un sacrifice, comme les marins le font dans le livre de Jonas, et, surtout, l'acquittement se concluait par un sacrifice de reconnaissance (tôdâh) complétant l'offrande votive. Le dernier sacrifice du nazir n'est pas appelé ainsi car il ne comporte pas de pain fermenté, mais il devait avoir la même fonction.

Le vœu de nazir, exprimant une plus grande piété que de simples vœux sans grande promesse, fut pratiqué sans évolution décelable à l'époque hellénistique et à l'époque romaine. Sa disparition s'explique par la fin des rites sacrificiels à Jérusalem après la destruction du temple, le nazir devant en effet trois sacrifices de bétail. Il fut donc un rite suivi mais non incontournable du judaïsme. Nous n'en avons d'ailleurs aucune mention dans les textes de Qumrân, pas même dans le Rouleau du Temple qui reprend pourtant les principaux rites décrits dans le Pentateuque. Seul un fragment (4Q472) mentionne le terme mais, curieusement, il ne s'agit pas d'une évocation du rite votif, le mot devant être compris littéralement: "les séparés », comme on lit dans la même phrase « les volontaires » et non «les nobles », nzyrym et ndybym étant apparemment « resémantisés ». Il semble bien que les deux termes soient appliqués aux membres de 
la communauté de Yachad très souvent mentionnée dans les rouleaux sectaires trouvés dans les grottes de Qumrân, qui n'étaient évidemment pas des nazirs, sinon au sens littéral du terme. S'il y a évocation de groupes de nazirs dans d'autres textes, c'est uniquement parce qu'ils venaient accomplir en même temps leur rite de fin de vœu au temple de Jérusalem, ils ne pouvaient former un groupe religieux à part comme les Esséniens. L'appartenance au groupe Yachad se faisait à la suite d'un serment, simple engagement sans contrepartie, très différent d'un vœu qui suppose le bienfait privé ou public. Les deux catégories étaient encore bien distinctes à l'époque romaine, leur seul point commun étant alors la promesse solennelle.

Quant aux exemples que l'on trouve dans les premiers écrits chrétiens, ils sont peu probants. Le vœu de Paul à Kenkhrées (Actes 18,18) ne correspond pas au rite de nazir de Nombres 6, pas plus qu'il ne correspond à celui de la Mishna même si bien des cas limites, sans grand rapport avec la réalité, sont discutés dans le traité. Le frère de Jésus, Jacques, qui fut à la tête de la communauté nazôréenne de Jérusalem, n'est pas dit nazir mais seulement « sanctifié dès le sein de sa mère », ne buvant ni vin ni boisson forte, le rasoir ne passant pas sur sa tête (Eusèbe de Césarée citant Hégésippe). À la différence de Actes 21, la source n'est pas ici Nombres 6 mais Juges 13, seul texte où le motif du nazir dès le sein est présent. Ce motif littéraire a d'ailleurs été repris bien avant par l'auteur des récits d'enfance de l'évangile de Luc afin d'embellir le récit de conception de Jean le Baptiste. Dans ce récit, si le texte-référence est bien celui de la conception de Samuel, Zacharie reprenant le rôle d'Hannah, il reste que le texte-source est Juges 13 avec la mention de «l'enfant grandissait et son esprit se fortifiait» (Luc 1, 80), phraséologie comparable à ce que l'on trouve au sujet de Samson : «le jeune enfant grandit. Et l'esprit de Yahweh commençait à l'agiter » (Juges 13, 24-25) - le motif est repris encore et développé pour Jésus (Luc 2, 40). Ainsi le terme et la notion de nazir furent étrangers aux premiers chrétiens qui n'ont fait qu'insérer un rite qu'ils croyaient être un vœu de purification et reprendre un motif littéraire déjà classique dans la littérature judéenne puisque Jérémie est consacré dès le ventre dans l'introduction hagiographique du 
livre du même nom. Ce n'est qu'au Ive siècle ap. J.-C. qu'Épiphane de Salamine, reprenant comme Eusèbe la notice d'Hégésippe sur Jacques, retrouve le terme de naziraios, afin de le distinguer notamment de nazôraios, et que Grégoire de Naziance polémique contre des chrétiens zélés de Cappadoce ayant consacré leur vie à Dieu et se faisant appeler Naziraioi.

Pour finir, Jésus ne fut évidemment pas nazir comme certains auteurs le supposent car le terme nazôraios (Nazôréen), qui le désigne parfois mais qui se rapporte plus vraisemblablement à l'origine à la communauté de Jérusalem avec son frère comme chef, est à distinguer de naziraios. Ils ne proviennent pas du même terme sémitique, le premier venant de nôçér et non de nâzîr , ce qui lui donnerait le sens d'« observant » ou plutôt de « gardien ».

$$
* * *
$$

Nous avons ajouté quelques pages proposant une lecture anthropologique des rites de cheveux. Rejetant les typologies habituelles, il nous a semblé plus pertinent d'interpréter ces rites non en fonction de leurs caractéristiques mais en fonction de leur finalité. Ceci nous a conduit à distinguer deux catégories opposées : des rites dominés par la notion d'offrande, pour lesquels seuls les cheveux sont coupés, et des rites à caractère apotropaïque ou purificatoire concernant également d'autres extrémités comme les poils et les ongles. Malgré l'aspect hybride et chargé idéologiquement du vœu de nazir judaïque, et même si les cheveux étaient détruits et non offerts, il nous a semblé qu'il appartenait plutôt à la première catégorie.

Pour prolonger l'étude du héros Samson, nous avons joint une analyse détaillée de l'histoire rédactionnelle de Juges 13-16, qui s'appuie sur une critique textuelle et littéraire du récit, tentant de dégager des figures successives du personnage : du géant mythique au juge «historique » en passant par le héros nazir. Nous avons restitué le récit à un stade intermédiaire : la première relecture yahwiste d'un géant proche du dieu solaire Shamash. Ce récit fut défini comme un mythe qui put à l'origine être la geste d'un dieu et n'être pas d'origine sémitique étant donné que Samson semble avoir un double hurrite dans la Bible même : Shamgar. Quoi qu'il 
en soit, même intégré dans l'histoire construite d'Israël, Samson est le seul personnage dont l'action reste intomporelle, donc mythique. Sa présence dans un corpus devenu la Bible a fait qu'il est devenu très rapidement un « juge grostesque », les exégètes d'aujourd'hui n'ayant pas plus de discernement que les auteurs d'antan (PseudoPhilon) en ne saisissant pas la nature hérö̈que de Samson.

À cela nous avons encore ajouté un tableau récapitulatif des indices trouvés en 1 Samuel qui permettent d'entrevoir un récit légendaire sur le roi Saül. Loin d'être un roi qui aurait failli à sa mission pour mieux intrôniser David, Saül était un jeune homme voué par sa mère nazir. Son principal mérite avait été de reprendre le coffre de Yahweh aux Philistins dans la ville de Gibea. La mise en évidence de tels indices nous a conduit à proposer une nouvelle approche de certains textes bibliques afin d'identifier des relectures successives pour parvenir au noyau originel d'un récit, historique ou non. Nous appuyant sur l'exemple célèbre de la double introduction du jeune David auprès du roi Saül, nous avons montré comment la critique littéraire peut résoudre les problèmes posés par la critique textuelle. En se dégageant de l'impératif textuel qui obligerait à choisir la version hébraïque massorétique ou la version grecque de la Septante, il est apparu que David intervint contre Goliath dans un récit limpide aux allures de conte héroïque, le David musicien du chapitre 16 étant ultérieur au David «frondeur». Autrement dit, aucune des deux versions n'est satisfaisante, le traducteur grec ayant probablement fait la critique littéraire de son texte hébraïque, cela afin d'éliminer les doublets. Et il fallait bien choisir entre les deux David pour être cohérent, surtout lorsqu'à la fin de 1 Samuel 17 Saül demande qui est ce jeune homme : comment ne pouvait-il pas reconnaître celui qui fut introduit auprès de lui dans le chapitre précédent? Le problème de la version grecque vient du fait que le traducteur a choisi le musicien et non le jeune berger, ce qui a rendu sa tâche presque impossible. La limpidité du récit se trouve donc dans la version hébraïque du chapitre 17, les doublets en moins, où l'on comprend pourquoi Saül demande qui est le jeune homme qui a terrassé le géant Goliath puisqu'il est venu de manière conjoncturelle sur le champ de bataille, pour approvisionner ses frères sur l'ordre 
de son père Jessé. Ce conte, qui précède le motif du David musicien, n'a évidemment rien d'historique, d'autant plus qu'il s'agit de la reprise d'un exploit d'un certain Elhanan mentionné en 2Samuel 21, 19, lui aussi de Bethléem (!), ayant vaincu le géant de Gat du même nom avec un simple bâton de bois dur. Cette pierre de remploi a donné donc bien des développements, qui la précèdent pourtant tous dans le livre ; bien souvent, ce qui est au début d'un livre ou d'un ensemble littéraire bibliques se trouve être la matière la moins ancienne.

Cette approche qui vise à isoler des pierres de remploi dans des « murs » textuels rénovés, comme c'est le cas pour le nazir dans le récit de Samson et surtout dans le texte reçu de la conception de Samuel, est ce que nous avons appelé une « archéologie des textes». Il ne s'agit pas véritablement d'une méthode mais d'une impulsion qui vise à inscrire plus encore les études bibliques dans les recherches historiques, archéologiques et anthropologiques du Proche-Orient ancien. Il nous semble que nous ne pouvons plus travailler sérieusement sur des textes qui ont été considérablement augmentés en ne prenant comme base de travail que le textus receptus et seulement ce texte-là. Le voyant anonyme de 1Samuel 9-10 indique bien à Saül qu'il doit aller à Gibea, les détours par Gilgal puis par Miçpa en rapport avec Samuel ne peuvent être pris en compte. Les incohérences littéraires sont telles parfois que même une lecture narratologique fine ne peut apporter un semblant de solution. Les textes bibliques sont composites et donc riches. À l'image de tells archéologiques, on a construit les nouvelles installations sur les anciennes. En outre, les éditeurs ont su garder parfois des pièces anciennes, juxtaposant un récit originel poétique à un récit relu et corrigé (Juges 4 et $5:$ le chant de Debora). Cherchons alors cette littérature ancienne qui se cache sous les couches deutéronomistes ou autres : il y a des fragments d'Iliade à trouver !

5 rue Malebranche

75005 Paris

ch.lemardele@laposte.net 